\title{
A Conceptual Framework for Computer- Supported Collaborative Learning at Work
}

\begin{abstract}
This paper identifies challenges for Computer-Supported Collaborative Learning at Work (CSCL@Work) by arguing that learning at work transcends our current understanding of learning as derived either from school learning or from current practices in adult education.

The paper develops a number of themes defining a conceptual framework for CSCL@Work including the following: distributed cognition, integration of problem framing and solving, communities of interest, and meta-design, cultures of participation, and social creativity as approaches to learning when the answer is not known. It illustrates the framework with two narratives, identifies some trade-offs and barriers, and briefly reflects on the impact and future of CSCL@Work.
\end{abstract}

Keywords CSCL, CSCW, distributed cognition, integration of problem framing and problem solving, communities of interest, learning when the answer is not known, meta-design, cultures of participation, social creativity, trade-offs and barriers

\section{Introduction}

Learning needs to be examined across the lifespan because previous notions of a divided lifetime - education followed by work - are no longer tenable [Gardner, 1991]. Professional activity has become so knowledge-intensive and fluid in content that learning has become an integral and essential part of adult work activities [Drucker, 1994]. "Learning is a new form of labor" (p 395) [Zuboff, 1988] and working is often (and must be) a collaborative effort among colleagues and peers. Knowledge, especially advanced knowledge, is acquired well past the age of formal schooling, and in many situations through educational processes that do not center on the traditional school [Collins \& Halverson, 2009; Illich, 1971; National-Research-Council, 2009]. Fundamentally new learning opportunities are required making computer-supported collaborative learning at work (CSCL@Work) a necessity rather than considering a luxury. I had two opportunities in the past to reflect upon situating CSCL@Work between the primary objectives of the $\mathrm{CSCW}$ and the CSCL research communities. In an invited lecture at ECSCW'2003 conference entitled "Working and Learning When the Answer is Not Known” (http://13d.cs.colorado.edu/ gerhard/presentations/ ecscw-keyn-slides-final.pdf) I argued that the CSCW community should be more concerned with learning for the following reasons:

- learning is an essential part of work, particularly in contexts where the answer is not known; 
- despite learning's important role, the CSCW community has largely neglected to use it as a theoretical and analytical construct; and

- future research challenges for CSCW should give more attention to learning, integrate working with learning and collaboration, and create innovative media to support this integration;

In an invited lecture at the CSCL'2007 conference entitled "Designing SocioTechnical Environments in Support of Meta-Design and Social Creativity" (http://13d.cs.colorado.edu/ gerhard/presentations/slides-cscl-final.pdf) I argued that the CSCL community should understanding learning in work environments and not just in schools and that many advanced learning technology approaches are too timid and are not thinking radically enough for the following reasons [Fischer, 2007]:

- they accept too many established approaches - e.g.: a theory of human learning based solely on school learning is too limited but the success of universal schooling has led us to identify learning with schooling;

- they insufficiently embrace new learning opportunities - e.g.: exploiting the unique opportunities of social production in which all learners can act as active contributors in personally meaningful problems;

- they reduce digital literacy and fluency to accessing and comprehending existing information rather than empowering learners to reformulate knowledge, to express themselves creatively and appropriately, and to produce and generate information; and

- they often do not move beyond "gift-wrapping" and "techno-determinism" to explore the co-evolution of learning, new media, and new learning organizations.

This paper is an elaboration of providing additional arguments about the importance and identity of CSCL@Work as a scientific discipline to rethink and reframe learning to account better for the demands of the knowledge societies of the future. The development of framework for CSCL@Work has been influenced by numerous other sources including:

- Illich's vision of "learning webs" conceiving a different style of learning, showing that "the inverse of school is possible", and defining educational resources (reference service to educators-at-large and to educational objects, skill exchange, peer matching) 40 years before the technological substrate made them possible [Illich, 1971].

- Resnick's differentiation of "learning in and out of schools" contrasting specifically: (1) individual cognition in school versus shared cognition outside; (2) pure mentation in school versus tool manipulation outside; (3) symbol manipulation in school versus contextualized reasoning outside school' and (4) generalized learning in school versus situation-specific competencies outside [Resnick, 1987].

- Engeström's concept of “expansive learning at work” grounding his approach in learning in work "people and organizations are all the time learning something that is not stable, not even defined or understood ahead of time. ... There is no competent teacher. Standard learning theories have little to offer if one wants to understand these processes" [Engeström, 2001]. 
The following sections will outline a conceptual framework (including the impact of new media and the articulation of research questions) and describe specific developments to addresses the challenges derived from the conceptual framework.

\section{Challenges for CSCL@Work}

Understanding "Work". The development of a conceptual framework for CSCL@Work requires some understanding of work and how it gets accomplished [Greenbaum, 1995; Orr, 1996]. Work covers an immense number of activities in an enormous number of application domains. This article focuses primarily on work that can be characterized as creative problem solving (in which processes are not well-defined) rather than on routine cognitive skills (in which processes are welldefined). For example: most design and decision making problems [Simon, 1996] (1) transcend the power of the unaided individual human mind requiring distributed cognition; (2) represent wicked, problematic situations rather than given problems requiring the integration of problem framing and problem solving; (3) are systemic problems requiring communities of interest consisting of participants with multiple expertise; and (4) represent problems to which the answers is not known requiring meta-design, cultures of participation, and social creativity. The "answer is not known" to these problems because design and decision making problems represent a universe of one supported by the evidence that "85 percent of the problems a doctor sees in his office are not in the book" [Schön, 1983].

CSCL@Work: In Search for a New Understanding of Learning. CSCL@Work is limited in what it can learn from most current approaches of CSCL which are primarily focused on school learning. Learning in schools is conceptualized primarily from the perspective that "answers are known" by teachers and learning is focused on the core objective that the students should learn as effectively as possible what the teachers know. This limitation has been articulated by a variety of researchers including Scribner and Sachs who assert: " $A$ decade of interdisciplinary research on everyday cognition demonstrates that school-based learning, and learning in practical settings, have significant discontinuities. We can no longer assume that what we discover about learning in schools is sufficient for a theory of human learning. " [Scribner \& Sachs, 1990]. Learning can no longer be dichotomized into a place and time to acquire knowledge (school) and a place and time to apply knowledge (the workplace) [Gardner, 1991]. Coverage of relevant subjects in schools is impossible and obsolescence is guaranteed.

The research in our Center for LifeLong Learning \& Design (L3D) (http://13d.cs.colorado.edu/) to make learning a part of life has been grounded in the exploration of the following basic assumption:

"If the world of working and living relies on collaboration, creativity, definition, and framing of problems, and if it requires dealing with uncertainty, change, and intelligence that is distributed across cultures, disciplines, and tools - then education should foster transdisciplinary competencies that prepare students for having meaningful and productive lives in such a world."

In addition to being limited what CSCL@Work can learn from learning in schools, it is also limited what it can learn from current practices in adult education and 
workplace learning [Resnick, 1987]. Workplace learning is often reduced to training by delivering courses that are separated from the contexts in which their subject matter is relevant [Fischer, 2000] and to knowledge management approaches which support only learning from the past [dePaula \& Fischer, 2005]. Lifelong learning provides a perspective for CSCL@Work with its basic assumption that learning is inherent in human nature, on ongoing and integral part of our lives, not a special kind of activity separable from the rest of our lives. From this perspective, learning, working, and collaborating are integrated and complement each other [Brown \& Duguid, 1991] to cope with the problems articulated in the next section.

\section{The Collaborative Aspect of CSCL@Work: Transcending the Individual}

Human Mind. CSCL@Work offers important possibilities to cope with major problems our societies are facing today and they can be classified as follows [Fischer, 2011]:

- problems of a magnitude which individuals and even large teams cannot solve (requiring support for distributed cognition);

- problems being poorly understood and wicked requiring the involvement of the owners of problems because they cannot be delegated to others (requiring support for the integration of problem framing and problem solving; see narrative 1 for illustration);

- problems of a systemic nature that do not fall within the boundaries of one specific domain (requiring the collaboration of many different minds from a variety of background in communities of interest); and

- problems modeling changing and unique worlds for which the answers are not known being dependent on open, living information repositories and tools (requiring support for end-user development and meta-design; see narrative 2 for illustration).

CSCL@Work and New Media. CSCL@Work can be facilitated and supported by a variety of different technological environments should not be reduced to

- "gift-wrapping" approaches that have been used primarily as add-ons to existing practices reducing the impact of new media to mechanize old ways of learning, working, and collaborating [Fischer, 2000];

- "technology-driven" developments that are grounded questionable assumption that new technologies are not only necessary, but also sufficient for causing fundamental changes towards a new culture of learning [Thomas \& Brown, 2011]

Innovative socio-technical environments for CSCL@Work should support the coevolution of a new understanding of learning, working, and collaborating, of new media and new technologies, and new learning organizations (see Figure 1). 


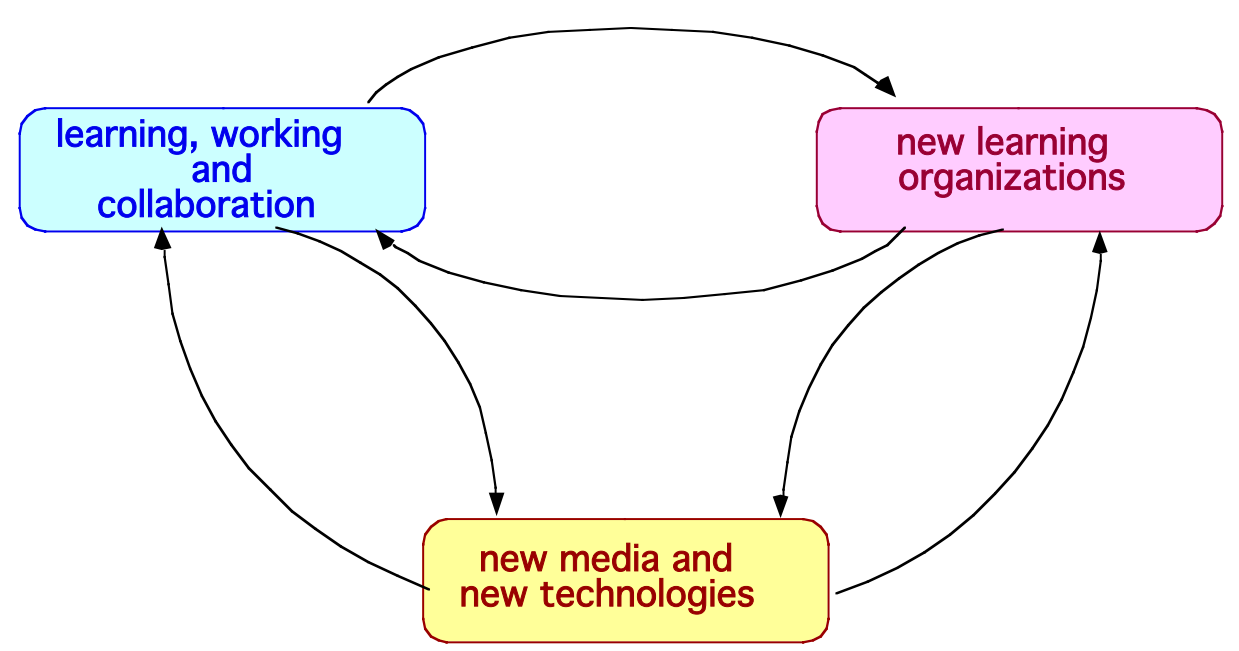

Figure 1: The Integration and Co-Evolution between Activities, Organizations, and Media

An example of gift-wrapping would be the use of students response systems (such as Clickers) to enhance classroom learning by supporting students to provide feedback and allowing teachers to get a quick assessment of the students' understanding. An example of technology-driven developments would be an objective such as "all schools on the Internet" (as promoted by Al Gore around 1994) which is necessary to explore different learning opportunities but not sufficient (without accompanied changes in content and pedagogy) to improve learning.

Research Questions (RQs). The preceding argumentation provides the foundation for the number of research questions (RQs) (first articulated in my presentation at the workshop "CSCL at Work" taking place at the GROUP'2010 conference) [Fischer, 2010]:

- RQ-1: Does distributed cognition provide an interesting perspective for CSCL@Work (based on the magnitude of problems)?

- RQ-2: Does the integration of problem framing and problem solving allow us to cope with poorly understood and ill-defined problems?

- RQ-3: Will communities of interests be more successful in coping with systemic problems than communities of practice?

- RQ-4: How do create frameworks, media, and organization to learn from each other when the answer is not known?

These research questions will be illustrated with two narratives and with developments of components of our conceptual framework in the following sections.

\section{Narrative-1: Writing this Article about CSCL@Work}

Writing an article like this one is an essential part of my work as a researcher. This brief narrative will briefly analyze and describe what this activity will contribute to the conceptual framework for CSCL@Work.

While I am the single author of this article, the work done is best understood from a distributed cognition perspective: I have (1) collaborated with many colleagues and students in developing the ideas and systems described (see the 
acknowledgements); (2) relied on many other sources documented in the literature (see the references); (3) built upon my own work of the past in developing these ideas (see introduction). The long term collaboration with ourselves has been characterized as reflexive $C S C W$ supporting the same individual user, who can be considered as two different persona at points of time that are far apart [Thimbleby et al., 1990]). I made extensive use of tools (which need to be learnt, used, and extended if necessary) such as taking advantage of a large, collaboratively constructed Endnote library, and my colleagues who provided me with feedback and criticism and facilitated this process with collaborative writing tools.

Going through many iterations of this article consisted not only of solving a problem, but led to numerous versions based on a reframing of the problem. The feedback for reflecting more deeply about the problem came for a community of interest: colleagues with different views of the problem working in the learning sciences, in school learning, in adult education, and in the development of new media. And least but not least: the answer to the problem "what is an interesting conceptual framework for CSCL@Work?" was not known — and the writing of this article represented learning, working, and collaborating to develop one argumentative context for the problem.

\section{The Conceptual Framework}

This section describes four components of a conceptual framework for CSCL@Work that we have explored in our research exploring the co-evolution between learning, working, and collaboration, new learning organizations, and new media (see Figure 1).

\section{Distributed Cognition}

CSCL@Work should be grounded in a distributed cognition framework because "people think in conjunction and partnership with others and with the help of culturally provided tools and implements" (p. xiii) [Salomon, 1993]. Distributed cognition [Hollan et al., 2001] is based on the assumption that knowledge does not reside solely in a person's head, but is created in social practices, and mediated by artifacts all situated in a socio-technical environment. The objective of workplace learning is to take as much as possible advantage of the knowledge that other people have and exploit the existence of powerful tools whereas school learning and its assessment [Resnick, 1987] is often focused on (1) the unaided mind by studying memory, attention, perception, action, and thought, unaided by external devices and other people; (2) on individual cognition: people are expected to learn and perform individually; and (3) demand for tool free performance (favoring tools for learning over tools for living). Creating externalizations [Bruner, 1996] (one of the main objectives of the EDC described in narrative 2) is of special importance for groups, because "a group has no head". For collaborative design activities, externalizations are a necessity because they create a record of our mental efforts, one that is "outside us" rather than vaguely in memory, and they represent situations which can talk back to us, critiqued, and negotiated [Fischer et al., 1998; Schön, 1983] 


\section{Integration of Problem Framing and Problem Solving}

Many problem-solving methodologies assume that problems can be clearly defined a priori - before any attempt at a solution is made. In work environments, as opposed to the classroom, problems and tasks themselves are ill-defined and wicked, and solution attempts represent moving targets requiring an integration of problem framing and problem solving [Rittel, 1984]. Work in progress as it manifests itself in externalizations, suggests ways to proceed, and the development of a solution causes the understanding of the task to grow and change.

Learning on demand [Fischer, 1991] often occurs in the context of integrating problem framing and problem solving as it represents an important opportunity to be exploited by reflection-in-action [Schön, 1983]. Learning on demand is the only viable strategy in a world where we cannot learn everything. It is a promising approach for the following reasons:

- it contextualizes learning by allowing it to be integrated into work rather than relegating it to a separate phase;

- it lets learners see for themselves the usefulness of new knowledge for actual problem situations, thereby increasing the motivation for learning new skills and information, and

- it makes new information relevant to the task at hand, thereby leading to better decision making, better products, and better performance.

In our own work we have pursued learning on demand in the context of developing conceptual frameworks and innovative systems for domain-oriented, programmable design environments [Eisenberg \& Fischer, 1994]. These environments explore middle ground between open learning environments (supporting self-directed learning opportunities but lacking guidance and support) and intelligent tutoring systems (providing extensive guidance and support but doing so for the problems irrelevant to the workers' tasks at hand). Domainoriented, programmable design environments support learner control, expressiveness, assistance, modifiability, domain-oriented descriptions, information delivery, contextualization of information to the task at hand, and collaboration between users and are particularly effective in exploiting the motivation of users by permitting learning to take place in the context of actual problem situations.

Learning on demand supports multiple learning strategies in response to the specific situation learners are facing: at certain times, they may be only interested in a quick fix or they want restrict their learning effort to reflect on a specific context, whereas in other situation they may want to explore a domain in a systematic fashion.

Table 1 describes three learning strategies with their respective strengths and weaknesses for workplace learning.

Table 1: Multiple Learning Strategies

\begin{tabular}{|l|l|l|l|}
\hline Level & Description & Strengths & Weaknesses \\
\hline $\begin{array}{l}\text { Fix-It } \\
\text { Level }\end{array}$ & $\begin{array}{l}\text { fixes the problem by } \\
\text { giving performance } \\
\text { support without detailed } \\
\text { understanding }\end{array}$ & $\begin{array}{l}\text { keeps focus on } \\
\text { task; learning does } \\
\text { not delay work }\end{array}$ & $\begin{array}{l}\text { creates little } \\
\text { understanding }\end{array}$ \\
\hline
\end{tabular}




\begin{tabular}{|l|l|l|l|}
\hline $\begin{array}{l}\text { Reflect } \\
\text { Level }\end{array}$ & $\begin{array}{l}\text { explores argumentative } \\
\text { context for reflection } \\
\text { ("reflection-in-action") }\end{array}$ & $\begin{array}{l}\text { understanding of } \\
\text { specific issues }\end{array}$ & $\begin{array}{l}\text { piecemeal } \\
\text { learning of } \\
\text { (disconnected) } \\
\text { issues }\end{array}$ \\
\hline $\begin{array}{l}\text { Tutorial } \\
\text { Level }\end{array}$ & $\begin{array}{l}\text { provides contextualized } \\
\text { tutoring (not lecturing on } \\
\text { unrelated issues) }\end{array}$ & $\begin{array}{l}\text { systematic } \\
\text { presentation of a } \\
\text { coherent body of } \\
\text { knowledge }\end{array}$ & $\begin{array}{l}\text { substantial time } \\
\text { requirements }\end{array}$ \\
\hline
\end{tabular}

\section{Communities of Interest}

Working on systemic problems requires the collaboration and coordination of stakeholders from different communities of practice (CoP) [Wenger, 1998]. We define a community of interest (CoI) [Fischer, 2005] as a group of stakeholders brought together from different $\mathrm{CoP}$, on the basis of a common concern or interest, to solve a particular complex design problem. In contrast to project teams, wherein employees are held together by a formal contract such as a business project, CoI stakeholders are held together by a shared interest.

CoIs are often more temporary than CoPs and can not rely on a shared social practice. Their raison d'être is a common interest in the framing and resolution of a design problem. They are less in anger to suffer from group-think [Janis, 1972] and can be more innovative and more transforming than CoPs if they can leverage on the "symmetry of ignorance" [Rittel, 1984] as a source for collective creative innovations. Challenges facing CoIs are in building a shared understanding of the problem at hand, which often does not exist at the beginning but evolves incrementally and collaboratively. Members of CoIs must learn to communicate with and learn from each other [Engeström, 2001], although they may have different perspectives and perhaps different vocabularies for describing their ideas. Learning within CoIs is more complex and multi-faceted than legitimate peripheral participation in CoPs [Lave \& Wenger, 1991] which assumes that there is a single knowledge system within which newcomers move toward the center over time. Acting knowledgeably in CoPs is less demanding compared to the challenges of operating within CoIs, which do not share a common language and practice. Strategies to mitigate these challenges and facilitate the sharing of knowledge and allowing knowledgeable performances within CoIs include: developing boundary objects [Bowker \& Star, 2000], engaging knowledge brokers, supporting transdisciplinary collaboration, and developing new media in support of these processes in order to circumvent the social and technical obstacles that often impede an effective exchange of information within CoIs and create new opportunities for social creativity.

\section{Learning when the answer is not known}

For many problems in work environments, the knowledge to understand, frame, and solve these problems does not already exist, but must be collaboratively constructed and evolved during the problem framing and solving process. Informed participation in a collaborative work setting requires information, but mere access to information is not enough. The participants must go beyond the information that 
exists to solve their problems. To support learning when the answer is not known, the primary role of media is not to deliver predigested information to individuals, but to provide the opportunity and resources for social debate, collaboratively created externalizations and the discussion and reflection about them. To transcend the existing information, tools, and artifacts and cope with the unique demands of problems, improving access to existing information (often seen as the major advance of new media) is a limiting aspiration. A more profound challenge is to allow stakeholders to incrementally acquire ownership in problems and contribute actively to their solutions. The following sections briefly describe three themes contributing to cope with this challenge:

- meta-design: empowers people to act as active contributors engaging in knowledge and tool creation [Fischer \& Giaccardi, 2006];

- cultures of participation: provide all people with the means to participate and to contribute actively in personally meaningful problems [Fischer, 2011]; and

- social creativity: distances, diversity, and emergence are important sources for social creativity and provide foundations for mutual learning when the answer is not known [Fischer et al., 2005].

Meta-Design. Meta-Design [Fischer \& Giaccardi, 2006] is focused on "design for designers." Meta-design is grounded in the basic assumption that highly creative owners of personally meaningful problems struggle and learn tools that are useful to them, rather than believing in the alternative of "ease-of-use," which limits them to preprogrammed features [National-Research-Council, 2003]. Users are empowered with opportunities, tools, and social rewards to extend a system to fit their needs at use time, rather than being forced to use closed systems created at design time [von Hippel, 2005]. As owners of problems, users can be active contributors engaged in creating knowledge rather than passive consumers restricted to the consumption of existing knowledge [dePaula \& Fischer, 2005]. Meta-design (1) supports a discourse focused on problem domains and not just the computer domain; (2) creates artifacts that can be subjected to critical reflection, open to adjustment and tweaking; (3) supports unintended and subversive uses (not just anticipated ones); and (4) allows learners to engage in personally meaningful activities.

Meta-design transcends the limitations of closed systems that do not give ownership to those who own the problem, but to a selected group of designers whose major challenge is to foresee all possible tasks and breakdowns in order to store answers to questions that might arise thereafter [Suchman, 1987; Winograd \& Flores, 1986]. Closed systems are likely to contain information that is chronically out of date and they are therefore not suited to the emerging idiosyncratic demands of workplace learning.

Cultures of Participation. Cultures are defined in part by their media and their tools for thinking, working, learning, and collaborating. In the past, the design of most media emphasized a clear distinction between producers and consumers [Benkler, 2006]. For example: instructionist, curriculum driven formal education treats learners as consumers, fostering a mindset in students of "consumerism" rather than "ownership of problems" for the rest of their lives [Illich, 1973]. As a result, learners, workers, and citizens often feel left out of decisions by teachers, managers, and policymakers, denying them opportunities to take active roles. 
The rise in social computing (based on social production and mass collaboration) has facilitated a shift from consumer cultures (specialized in producing finished artifacts to be consumed passively) to cultures of participation (in which all people are provided with the means to participate and to contribute actively in personally meaningful problems) [Fischer, 2011; Jenkins, 2009]. These developments represent unique and fundamental opportunities, challenges, and transformative changes for CSCL@Work as we move away from a world in which a small number of people define rules, create artifacts, make decisions for many consumers towards a world in which everyone has interests and possibilities to actively participate.

Fostering cultures of participation is supported by meta-design by creating the following mechanisms:

- Making changes must seem possible: Contributors should not be intimidated and should not have the impression that they are incapable of making changes; the more users become convinced that changes are not as difficult as they think they are, the more they may be willing to participate.

- Changes must be technically feasible: If a system is closed, then contributors cannot make any changes; as a necessary prerequisite, there needs to be possibilities and mechanisms for extensions.

- Benefits must be perceived: Contributors have to believe that what they get in return justifies the investment they make. The benefits perceived may vary and can include: professional benefits (helping for one's own work), social benefits (increased status in a community, possibilities for jobs), and personal benefits (engaging in fun activities).

- Sharing changes must be encouraged: evolutionary growth is greatly accelerated in systems in which participants can share their contributions without substantial additional efforts

- Meta-designers must exist and be rewarded: meta-designers should use their own creativity to create socio-technical environments in which other people can be creative. They must create contexts in which users acting as designers can create content and they must be willing to share control about tools and content.

Social Creativity. Although creative individuals are often thought of as working in isolation, much human creativity arises from activities that take place in a social context in which interaction with other people and the artifacts that embody collective knowledge are important contributors to the process [Csikszentmihalyi, 1996]. As argued before: the fundamental problems of the $21^{\text {st }}$ century are complex and open-ended, requiring ongoing contributions of many minds, particularly from the people who "own" the problems and are directly affected by them.

CSCL@Work should provide frameworks to invent alternative social organizations and new media that will permit the flourishing of deep interdisciplinary specialties [Derry et al., 2005], as argued for by Campbell [Campbell, 2005]: "Even within disciplines, disciplinary competence is not achieved in individual minds, but as a collective achievement made possible by the overlap of narrow specialties." The fish-scale model (trying to achieve "collective comprehensiveness through overlapping patterns of unique narrowness") proposed by Campbell provides a viable path toward a new competence, based on the integration of individual and social creativity [Fischer et al., 2005]. 
Distances (across spatial, temporal, and technological dimensions) and diversity (bringing stakeholders together from different cultures in CoIs) are important sources for social creativity [Fischer, 2005]. Over the last decade, we have designed and developed socio-technical environments exploring and supporting different aspects of social creativity including (1) the Envisionment and Discovery Collaboratory described in Narrative-2; (2) the CreativeIT Wiki to support the research community in "Creativity and IT"; and (3) collaborated with Google in analyzing the $3 D$ Warehouse (one component of interconnected tools that include SketchUp and Google Earth), which stores user-generated 3D models of buildings (and other artifacts) contributed from participants distributed all over the world. In these contexts, we have studied different aspects of social creativity, including the impact of cultures of participation, motivation to contribute, learning requirements to become a contributor, and the role of curators to organize the emerging large repositories.

\section{Narrative-2: Framing and Solving Urban Planning Problems with the Envisionment and Discovery Collaboratory}

The Envisionment and Discovery Collaboratory (EDC) [Arias et al., 2000] represents a new generation of collaborative domain-oriented design environments. It shifts the emphasis away from the computer screen as the focal point and creates an immersive environment in which stakeholders can incrementally create a shared understanding. It attempts to maximize the richness of communication between stakeholders in face-to-face interaction, mediated by both physical and computational objects. It is grounded in Schön's "reflection-in-action" problemsolving approach [Schön, 1983] and has been applied to a variety of different design and decision making problems in urban planning, emergency management, and transportation planning. Stakeholders using the EDC (see Figure 2) convene around a computationally enhanced table that serves as the action space. The action space allows users to manipulate an interactive model of the design problem by proposing, exploring, reflecting, and critiquing alternative solutions in a complex design space. The table is flanked by another touch-sensitive (vertical) surface that serves as the reflection space. The reflection space displays information that is relevant to the context as defined by the simulation in the action space. 


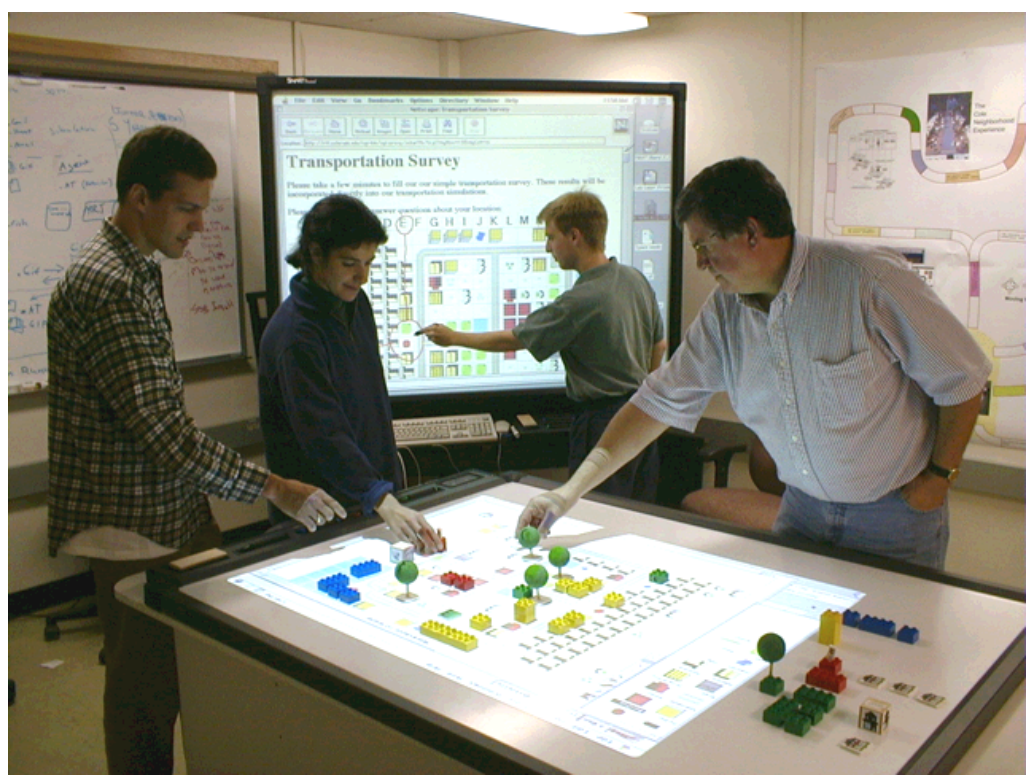

Figure 2: The Envisionment and Discovery Collaboratory

The EDC explores and instantiates the components of our conceptual framework for CSCL@Work in a variety of different dimensions:

Distributed cognition is facilitated and enhanced by a table-top computing environment that supports groups of people to work and learn together and contains substantial information and tools relevant to the domain under investigations.

The integration of problem framing and problem solving is supported by a linkage between an action space (see Figure 3) and an reflection space (see Figure 4). When the participating stakeholders consider how land should be used in planning a new urban environment, they deal with a complex and wicked situation in which geographic, topological, financial, economic, and political issues are all mixed up together. During their collaborative activities in defining a land use scheme in the action space, they can analyze and reflect upon their decision making at any time with an alternative view of their action in the reflection space providing them with a quantitative distribution. Going back and forth between the two spaces provides the participants with numerous learning possibilities. The transition from the action to the reflection space is often triggered by critics [Fischer et al., 1998] that signal a breakdown situation such as the violation of existing knowledge. 


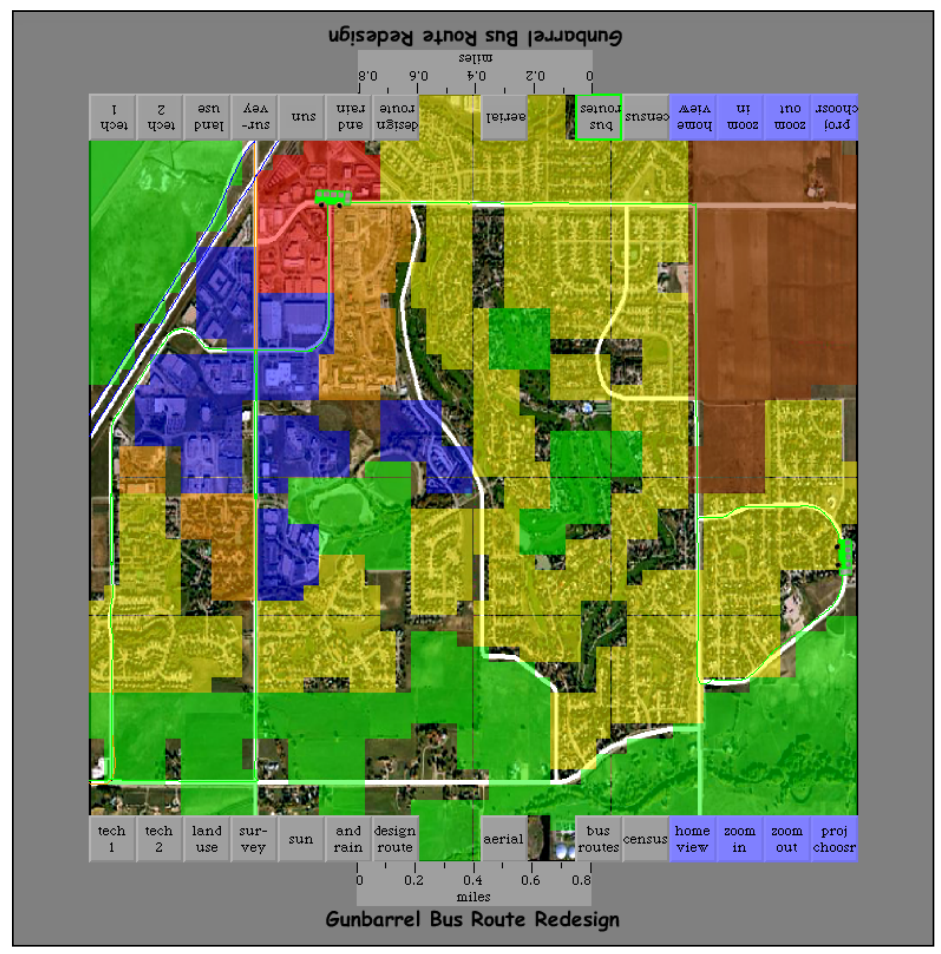

Figure 3: A Land-Use Scenario in the Action Space

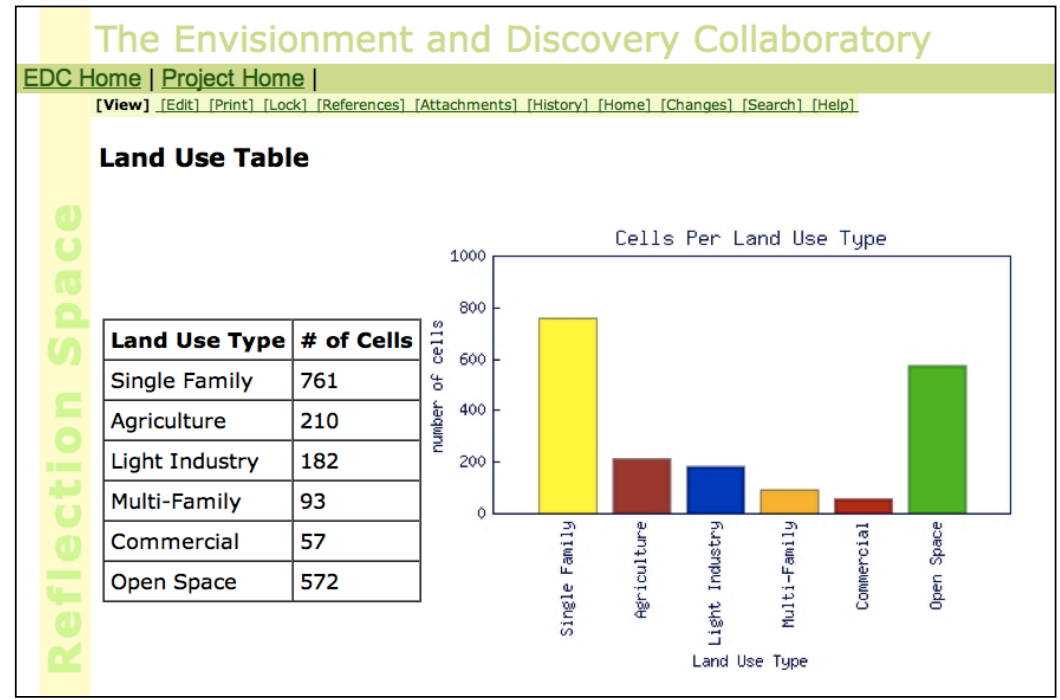

Figure 4: Summary View of Land Use Generated in the Reflection Space

Communities of Interest: the EDC brings together stakeholders with different expertise, including professional planners, financial experts, transportation planners, and financial experts. It exploits the symmetry of ignorance (i.e., that all involved stakeholders can contribute actively) as a source of power for mutual learning by providing all stakeholders with means to express their ideas and their concerns.

Learning when the answer is not known: the three themes identified to cope with this objective are supported by the EDC as follows: 
- meta-design: the EDC was envisioned to be a end-user modifiable version of Simcity, allowing participants to contextualize the existing environment to their own situation, integrate different information sources, and develop new structures and processes;

- cultures of participation: the EDC is an environment that is not restricted to the delivery of predigested information to individuals, but it provides opportunities and resources for design activities embedded in social debates and discussions in which all stakeholders can actively contribute rather than being confined to passive consumer roles;

- social creativity: the EDC supports emerging insight as illustrated by the following example in which participants collaboratively try to decide the best location of a bus stop by analyzing multiple walking distances (see Figure 5). Participants indicate where they live on the map (resulting in a house icon appearing at that location). They are further supported with taskfocused interaction mechanisms to indicate how far they would be willing to walk in good and bad weather. After specifying this information, colored circles (generated by the system) appear around their individual house icons, indicating the range of area that they might be willing to walk to catch a bus. The display (bringing together the results of their individual decisions) shows emerging, overlapping patterns of areas that are suitable for bus routes and bus stops, providing information and perspectives that no individual had in her of his head prior to the exercise. This externalization created by individual actions serves as a source of social creativity for further design and decision making.

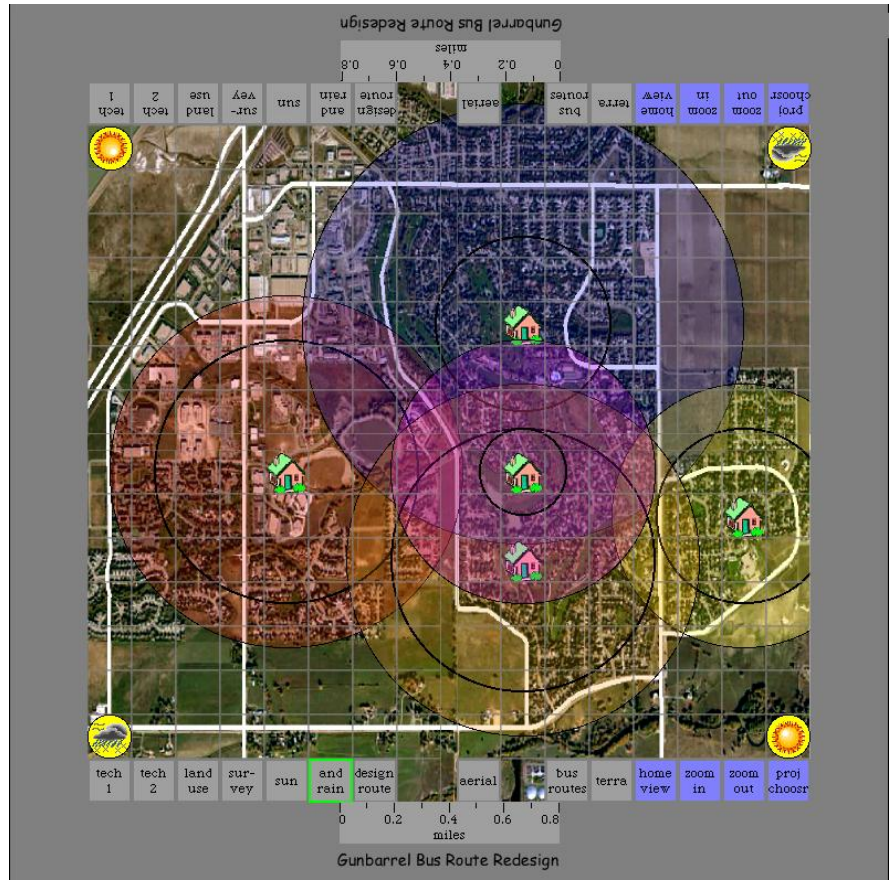

Figure 5: Emerging Collaborative Insights based on Individual Contributions in a WalkingDistance Scenario 


\section{Trade-Offs and Barriers of CSCL@Work}

Being Taken of Task. A researcher needs to finish a paper facing an upcoming deadline when her graduate student informs her that there are much improved versions of the tools (for writing, graphics, and creating references) available. She then face the production paradox [Carroll \& Rosson, 1987]: how to balance effort and time to get the work done (finishing the article) and do so with suboptimal tools versus engage in learning to use the new tools. The dilemma of the active user is related to the rational choices workers make while facing competing or conflicting situations, such as the trade-offs between dealing with pressing problems and investing in long-term solutions (such as learning to use a new technology).CSCL@Work may be able to address this paradox by allowing users to choose different strategies (such as: Fix-It, Reflect, or Tutorial; see Table 1) for learning something new depending on the different commitments. Another way to tackle the production paradox is to better integrate learning and working for any activity in a work practice. Toward this end, innovations in the workplace have to be meaningfully integrated into practices, so that learning and use become the same activity through which users can see tangible benefits and long-term impact in their work practices and careers.

\section{Learning on Demand: Making Information Relevant to Users and Tasks} versus Serendipity. Information access and information delivery are two approaches for obtaining information. Information access is a user-initiated search, while information delivery is a system-initiated presentation of information. In information access schemes, workers articulate information needs, while in information delivery schemes, systems infer information needs. Information delivery is particularly important when workers are not motivated to look for information or when they are not aware of the need for information in the first place.

System developments for CSCL@Work face another interesting paradox: whether to make information relevant to users and tasks or whether to facilitate serendipity. In a world of information overload, throwing decontextualized information at users (as in broadcast systems or in tool such as "Microsoft's "Tip of the Day") is of little use. Models of learners and task (for which information is readily available in today's computational environment from all sources) can be used to tailor information: a blessing to bombard users with less relevant information but taking away control from users what they want to see ([Pariser, 2011] characterizes this as a shift from human gate-keepers to algorithmic gate-keepers leading to isolation in a "web of one").

Tools for Learning and Tools for Living. In our research to create socio-technical environments to support caregivers and persons with cognitive disabilities, we have identified and explored a fundamental distinction in how we think about the empowerment and augmentation of human beings and the change of tasks in a toolrich world by identifying two major design perspectives [Carmien et al., 2007]:

- Tools for Living: grounded in a "distributed cognition" perspective, in which intelligence is mediated by tools for achieving activities that would be error prone, challenging, or impossible to achieve

- Tools for Learning: grounded in a "scaffolding with fading" perspective leading to autonomous performance by people without tools. 
This distinction raises fundamental questions about future of education, learning, working, and the development and use of new media by asking what it means to learn in the 21 st century in which powerful tools are available for many intellectual activities - allowing people to have instant access to facts, assisting people in spelling, doing arithmetic, and performing numerous other intellectual activities [Pea, 2004]. Therefore, our research makes fundamental contributions not only to a conceptual framework for designing more effective assistive technologies, but also to the future of education, learning, and the development and use of new media in general [Bransford et al., 2001].

"Build It and No One Comes". One major challenge in the design and deployment of collaborative systems relying on cultures of participation is that "we build it, and no one comes" [Smith \& Farquhar, 2000]. Collaborative and evolving systems are of no value or use without users' active and informed participation and contributions.

\section{The Impact and The Future of CSCL@Work}

A major impact of CSCL@Work will be to conduct critical research on the monopoly and exclusiveness of formal school learning. CSCL@Work will also complement and enrich current adult education that often is reduced to training based on a transmission model of learning. Inspirations, concepts, and organization may come from analyzing cultures of participation, in which people collaboratively learn and share their activities and insights and from the right kind of games [Reeves \& Read, 2009] focused on providing feedback in support of reflection-inaction, collaboration between players, player extensions, and engagement and flow [Csikszentmihalyi, 1996].

CSCL@Work provides the unique opportunity to explore and define conceptually alternative modes of computer-supported, collaborative learning that will enrich learning and working in the $21^{\text {st }}$ century. It has the potential to close the gap between school and workplace learning by allowing learners to engage in activities requiring collaboration, creativity, problem framing, and distributed cognition. It will provide us with insights and alternative models by illustrating what can be what must be learned as a result of intentional teaching and what can be learnt from working on interesting problems with others humans and with powerful innovative computational media.

\section{Acknowledgments}

I thank the members of the Center for LifeLong Learning \& Design at the University of Colorado, who have made major contributions to the ideas and developments described in this paper. Hal Eden and Ernesto Arias deserve special thanks for their development of the EDC that has served as an important object-tothink-with for this article. The research was supported in part by the (1) National Science Foundation IIS-0613638, IIS-0843720, IIS-0968588, OCI-1028017, (2) Google, and (3) SAP. I am grateful to Sean Goggins and Isa Jahnke (1) for providing me with the opportunity to present a keynote lecture in their workshop "CSCL@Work" at the 
GROUP 2010 conference that provided an important seed for this article, and (2) for constructive feedback to earlier version of this article.

\section{References}

Arias, E. G., Eden, H., Fischer, G., Gorman, A., \& Scharff, E. (2000) "Transcending the Individual Human Mind-Creating Shared Understanding through Collaborative Design," ACM Transactions on Computer Human-Interaction, 7(1), pp. 84-113.

Benkler, Y. (2006) The Wealth of Networks: How Social Production Transforms Markets and Freedom, Yale University Press, New Haven.

Bowker, G. C., \& Star, S. L. (2000) Sorting Things out - Classification and Its Consequences, MIT Press, Cambridge, MA.

Bransford, J. D., Brown, A. L., \& Cocking, R. R. (Eds.) (2001) How People Learn - Brain, Mind, Experience, and School, National Academy Press, Washington, D.C.

Brown, J. S., \& Duguid, P. (1991) "Organizational Learning and Communities-of-Practice: Toward a Unified View of Working, Learning, and Innovation," Organization Science, 2(1), pp. 40-57.

Bruner, J. (1996) The Culture of Education, Harvard University Press, Cambridge, MA.

Campbell, D. T. (2005) "Ethnocentrism of Disciplines and the Fish-Scale Model of Omniscience." In S. J. Derry, C. D. Schunn, \& M. A. Gernsbacher (Eds.), Interdisciplinary Collaboration - an Emerging Cognitive Science, Lawrence Erlbaum, Mahwah, NJ, pp. 3-21.

Carmien, S., Kollar, I., Fischer, G., \& Fischer, F. (2007) "The Interplay of Internal and External Scripts." In F. Fischer, I. Kollar, H. Mandl, \& J. M. Haake (Eds.), Scripting Computer-Supported Learning - Cognitive, Computational, and Educational Perspectives, Springer, New York, NY, pp. 303-326.

Carroll, J. M., \& Rosson, M. B. (1987) "Paradox of the Active User." In J. M. Carroll (Ed.), Interfacing Thought: Cognitive Aspects of Human-Computer Interaction, The MIT Press, Cambridge, MA, pp. 80-111.

Collins, A., \& Halverson, R. (2009) Rethinking Education in the Age of Technology: The Digital Revolution and the School, Teachers College Press New York, NY.

Csikszentmihalyi, M. (1996) Creativity - Flow and the Psychology of Discovery and Invention, HarperCollins Publishers, New York, NY.

dePaula, R., \& Fischer, G. (2005) "Knowledge Management: Why Learning from the Past Is Not Enough!" In J. Davis, E. Subrahmanian, \& A. Westerberg (Eds.), Knowledge Management: Organizational and Technological Dimensions, Physica Verlag, Heidelberg, pp. 21-54.

Derry, S. J., Schunn, C., \& Gernsbacher, M. A. (Eds.) (2005) Interdisciplinary Collaboration: An Emerging Cognitive Science, Erlbaum, Mahwah, NJ.

Drucker, P. F. (1994) "The Age of Social Transformation," The Atlantic Monthly(November), pp. 53-80.

Eisenberg, M., \& Fischer, G. (1994) "Programmable Design Environments: Integrating EndUser Programming with Domain-Oriented Assistance." In Human Factors in Computing Systems, Chi'94 (Boston, Ma), ACM, New York, pp. 431-437.

Engeström, Y. (2001) "Expansive Learning at Work: Toward an Activity Theoretical Reconceptualization," Journal of Education and Work, 14(1), pp. 133-156.

Fischer, G. (1991) "Supporting Learning on Demand with Design Environments." In L. Birnbaum (Ed.), International Conference on the Learning Sciences (Evanston, Il), Association for the Advancement of Computing in Education, pp. 165-172.

Fischer, G. (2000) "Lifelong Learning - More Than Training," Journal of Interactive Learning Research, Special Issue on Intelligent Systems/Tools In Training and Life-Long Learning (eds.: Riichiro Mizoguchi and Piet A.M. Kommers), 11(3/4), pp. 265-294.

Fischer, G. (2005) "Distances and Diversity: Sources for Social Creativity." In Proceedings of Creativity \& Cognition, ACM, London, April, pp. 128-136. 
Fischer, G. (2007) "Designing Socio-Technical Environments in Support of Meta-Design and Social Creativity." In Proceedings of the Conference on Computer Supported Collaborative Learning (CSCL '2007), Rutgers University, July, pp. 1-10.

Fischer, G. (2010) Challenges and Conceptual Frameworks for Computer-Supported Learning (CSCL) at Work. Presented at ACM Conference Group 2010, Workshop "CSCL at Work". available at http://www.csclatwork.org/node/9

Fischer, G. (2011) "Understanding, Fostering, and Supporting Cultures of Participation," ACM Interactions XVIII.3 (May + June 2011), pp. 42-53.

Fischer, G., \& Giaccardi, E. (2006) "Meta-Design: A Framework for the Future of End User Development." In H. Lieberman, F. Paternò, \& V. Wulf (Eds.), End User Development, Kluwer Academic Publishers, Dordrecht, The Netherlands, pp. 427457.

Fischer, G., Giaccardi, E., Eden, H., Sugimoto, M., \& Ye, Y. (2005) "Beyond Binary Choices: Integrating Individual and Social Creativity," International Journal of HumanComputer Studies (IJHCS) Special Issue on Computer Support for Creativity (E.A. Edmonds \& L. Candy, Eds.), 63(4-5), pp. 482-512.

Fischer, G., Nakakoji, K., Ostwald, J., Stahl, G., \& Sumner, T. (1998) "Embedding Critics in Design Environments." In M. T. Maybury, \& W. Wahlster (Eds.), Readings in Intelligent User Interfaces, Morgan Kaufmann, San Francisco, pp. 537-559.

Gardner, H. (1991) The Unschooled Mind, BasicBooks, New York.

Greenbaum, J. (1995) Windows on the Workplace: Computers, Jobs, and the Organization of Office Work in the Late Twentieth Century, Monthly Review Press, New York.

Hollan, J., Hutchins, E., \& Kirsch, D. (2001) "Distributed Cognition: Toward a New Foundation for Human-Computer Interaction Research." In J. M. Carroll (Ed.), Human-Computer Interaction in the New Millennium, ACM Press, New York, pp. 7594.

Illich, I. (1971) Deschooling Society, Harper and Row, New York.

Illich, I. (1973) Tools for Conviviality, Harper and Row, New York.

Janis, I. (1972) Victims of Groupthink, Houghton Mifflin, Boston.

Jenkins, H. (2009) Confronting the Challenges of Participatory Cultures: Media Education for the 21st Century, MIT Prress, Cambridge, MA.

Lave, J., \& Wenger, E. (1991) Situated Learning: Legitimate Peripheral Participation, Cambridge University Press, New York.

National-Research-Council (2003) Beyond Productivity: Information Technology, Innovation, and Creativity, National Academy Press, Washington, DC.

National-Research-Council (2009) Learning Science in Informal Environments - People, Places, and Pursuits, National Academy Press, Washington, DC.

Orr, J. (1996) Talking About Machines - an Ethnography of a Modern Job, ILR Press/Cornell University Press, Ithaca.

Pariser, E. (2011) Beware Online "Filter Bubbles" (Ted Video), available at http://www.youtube.com/watch?v=B8ofWFx525s.

Pea, R. D. (2004) "The Social and Technological Dimensions of Scaffolding and Related Theoretical Concepts for Learning, Education, and Human Activity," The Journal of the Learning Sciences, 13(3), pp. 423-451.

Reeves, B., \& Read, J. L. (2009) Total Engagement: Using Games and Virtual Worlds to Change the Way People Work and Business Compete, Havard Business Press, Boston, MA.

Resnick, L. B. (1987) "Learning in School and Out," Educational Researcher, 16(9), pp. 13-20.

Rittel, H. (1984) "Second-Generation Design Methods." In N. Cross (Ed.), Developments in Design Methodology, John Wiley \& Sons, New York, pp. 317-327.

Salomon, G. (Ed.) (1993) Distributed Cognitions: Psychological and Educational Considerations, Cambridge University Press, Cambridge, United Kingdom.

Schön, D. A. (1983) The Reflective Practitioner: How Professionals Think in Action, Basic Books, New York.

Scribner, S., \& Sachs, P. (1990) "On the Job Training: A Case Study." In National Center on Education and Employment, pp. 1-4. 
Simon, H. A. (1996) The Sciences of the Artificial, third ed., The MIT Press, Cambridge, MA.

Smith, R. G., \& Farquhar, A. (2000) "The Road Ahead for Knowledge Management — an Ai Perspective," AI Magazine, 21(4), pp. 17-40.

Suchman, L. A. (1987) Plans and Situated Actions, Cambridge University Press, Cambridge, UK.

Thimbleby, H., Anderson, S., \& Witten, I. H. (1990) "Reflexive CSCW: Supporting LongTerm Personal Work," Interacting with Computers, 2(3), pp. 330-336.

Thomas, D., \& Brown, J. S. (2011) A New Culture of Learning: Cultivating the Imagination for a World of Constant Change, CreateSpace, Lexington, KY.

von Hippel, E. (2005) Democratizing Innovation, MIT Press, Cambridge, MA.

Wenger, E. (1998) Communities of Practice - Learning, Meaning, and Identity, Cambridge University Press, Cambridge, UK.

Winograd, T., \& Flores, F. (1986) Understanding Computers and Cognition: A New Foundation for Design, Ablex Publishing Corporation, Norwood, NJ.

Zuboff, S. (1988) In the Age of the Smart Machine: The Future of Work and Power, Basic Books, New York. 